\title{
New Design of Model Reference Adaptive Control Systems
}

\section{P R V Rao*}

School of Engineering, University of Ulster at Jordamstown, Newotownabbey, Belfast BT37 OQB, United Kingdom

\begin{abstract}
This paper presents improved new adaptive laws in the design of Model Reference Adaptive Control (MRAC) systems, based on Lyapunov stability theory.It focuses on the standard adaptive laws which were developed based on a Positive Definite Quadratic Lyapunov functions; these standard laws make the derivative of the Lyapunov function only negative semi-definite; this feature was observed for the past three decades (1970s, 80s and 90s). However, it is noted that the transient response of the system error converging to zero has been significantly oscillatory. It is in this context, this paper presents new improved adaptive laws using a new Lyapunov function that employs the integral of square of error signal. The new adaptive laws offer improved transient response of the system error converging to zero-in particular, in a less oscillatory fashion. In addition, these new adaptive laws lead the derivative of the new Lyapunov function to become negative definite, along as the system error is not zero. The paper presents first mathematical development for $1^{\text {st }}$ order and $2^{\text {nd }}$ order systems, having relative degree as unity; then, it later presents simulation study, using the MATLAB package. The simulation results support the mathematical arguments.
\end{abstract}

Keywords: Reference Adaptive Control; Quadratic Lyapunov Functions; Adaptive Laws

\section{Introduction}

In systems and control engineering, and in real practical situation suggests how the existing theory needs to be modified to accommodate more realistic conditions on real plants. Much research has been carried out in this area of designing controllers for real plants. Controller design is made easer by the availability of information on the real plant (model structure, values of the plant parameters and acting disturbances are well known); so it is straightforward to obtain a desired behaviour of the plant output. Model Reference Adaptive Control Scheme [1]: With earlier Adaptive Laws when the plant model structure is known but its parameters are unknown, the process of developing a control scheme naturally becomes complicated. In general, it has been a common experience to find that more complex is the plant model: the more complex is the design of a suitable controller scheme.

Adapting/adjusting the controller parameters for such cases (where plant parameters were not accurately known), is known as 'Adaptive Control'. A typical adaptive controller scheme has mechanism in an additional loop (over and above the ordinary feedback loop) for adjusting/adapting the controller parameters. The equations, which describe how these controller parameters need to be adjusted, are called 'Adaptive Laws'. Since the overall system stability is the primary requirement, adaptive control schemes and adaptive laws have been developed based on Lyapunov stability theory, historically since the 1960s.

The new laws in this paper improve the overall system stability and offer optimal tuning for controller parameters, but also they are able to provide better dynamic performance and faster decay of system output error signal.

\section{Model reference adaptive control}

The main purpose of adaptive control is to control of systems which have parameters that are not accurately known. This inaccuracy can arise from changes in environment, such as temperature in thermal systems, density of air or water as in flying vehicles or submarines. Some system changes can be unpredictable, and ordinary closed-loop systems may not respond properly when the system transfer function varies. In many such circumstances, adaptive control is justified. Among many approaches for adaptive control of such systems, the Model Reference Adaptive Control has become a popular and widely accepted approach for incorporating the adaptive feature into an ordinary feedback control system. It was developed during 1960s. A general scheme of MRAC is shown in Figure 1.

In MRAC, a reference model is apriority chosen so that it describes the desired response of the plant controller combination; this choice is made by the designer, based on experience. The plant output is compared with the reference model output; the difference between them, called a system output is used to adjust the controller parameters such a way until this very error signal is driven to zero.In this MRAC, two features play crucial role: 1) Stability of the overall system, including the adaptive mechanism-in the sense that all signals in the entire system remain bounded; and 2) The plant output response meeting the reference model output response, with minimum error;

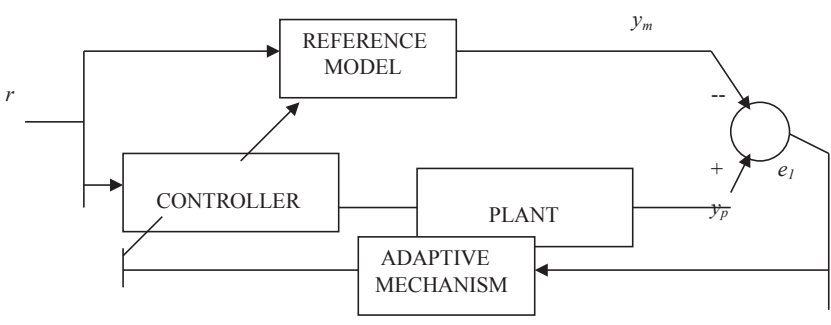

Figure 1: General Scheme of MRAC.

${ }^{*}$ Corresponding author: MPRV Rao, School of Engineering, University of Ulster at Jordamstown, Newotownabbey, Belfast BT37 0QB, United Kingdom, E-mail: musgrnc@erciyes.edu.tr

Received October 07, 2013; Accepted December 30, 2013; Published January 10, 2014

Citation: Rao MPRV (2014) New Design of Model Reference Adaptive Control Systems. J Appl Mech Eng 3: 134. doi:10.4172/2168-9873.1000134

Copyright: @ 2014 Rao MPRV. This is an open-access article distributed under the terms of the Creative Commons Attribution License, which permits unrestricted use, distribution, and reproduction in any medium, provided the original author and source are credited. 
that is, the system output error converging to zero. Consequently, after an MRAC scheme is designed, the entire system is simulated to check whether the above two feature are satisfied.

The MRAC has basically four blocks: 1) Plant, 2) Controller, 3) Reference Model, and 4) The Adaptive mechanism that adjusts the controller parameters. The design process involves setting up a controller (with adjustable parameters) with a structure that matches the structure of the given plant; choosing a stable reference model that represents the desired dynamic response of the plant controller combination; and, finally, developing a stable adaptive mechanism that adjusts the controller parameters. The equations which describe how exactly the controller parameters need to be changed are called adaptive laws. The design of stable MRAC has come to mean mainly the design of adaptive mechanism which, in turn, has come to mean the design of stable adaptive laws for adjusting the controller parameters.

\section{This paper is organised as follows.}

Section 2 describes the study on for $1^{\text {st }}$ order MRAC systems: Section 3 describes the study on for $2^{\text {nd }}$ order MRAC systems: it first provides mathematical development and simulations results of earlier standard adaptive laws based on Positive Definite Quadratic (PDQ) Lyapunov functions; later, it presents mathematical development. Section 4 presents simulation study. Finally, section 5 presents a conclusion.

\section{MRAC scheme for a 1storder plant}

For ease of exposition, consider the MRAC scheme with the controller a simple feedback gain parameter. $\theta$; The reference model be a first order system with a unity gain and a constant parameter; and the plant is a first order system with a unity and a constant parameter $a_{p}$.

$$
\text { Plant: } y_{p}=-\vartheta y_{p}+r \text {; Reference Model: } y_{m}=-\mathrm{a}_{\mathrm{m}} y_{m}+r
$$

Let the system output error elbe defined as the difference between plant output $y_{p}$ and reference model output $y_{p}$. Then, for the ideal controller parameter $\theta^{*}$, the plant controller combination becomes the reference model. For non-ideal values of $\theta$, there exists a parameter error, say, $\phi$ given by $\phi=\theta-\theta^{\star}$. In this scenario, the design of MRAC or development of adaptive law for adjusting the $\phi$ or $\theta$ based on Lyapunov stability theory can be outlined as follows.

The system output error equation can be written as

$$
\dot{e}_{1}=-e_{1}+\phi y_{p} \text { for } a_{1}=1
$$

Then, for the standard adaptive law, the positive definite quadratic Lyapunovfunction [2] is chosen as

$$
V=\frac{1}{2}\left[e_{1}^{2}+\phi^{2}\right]
$$

This function $V$ is differentiated with respect to time, system error equation is substituted and the standard adaptive law is chosen as shown in Figure 2

$$
\dot{\phi}=\dot{\theta}=-[g] e_{1} y_{p} \text { so that the derivative of } V \text { becomes, } \dot{V}=-a_{m} e_{1}^{2} \leq 0 \text { (4) }
$$

where gis a positive constant (say $g=1$ ). Now, for this standard adaptive law as shown in Figure 3, stability is assured as follows:since the $V$ is chosen as positive definite and the derivative of $V$ is obtained as negative semi-definite, the error system defined by the system output error equation and the adaptive law is uniformly stable. For details, see Narendra and Annaswamy [3].

Where as the work in this project presents a new improved standard

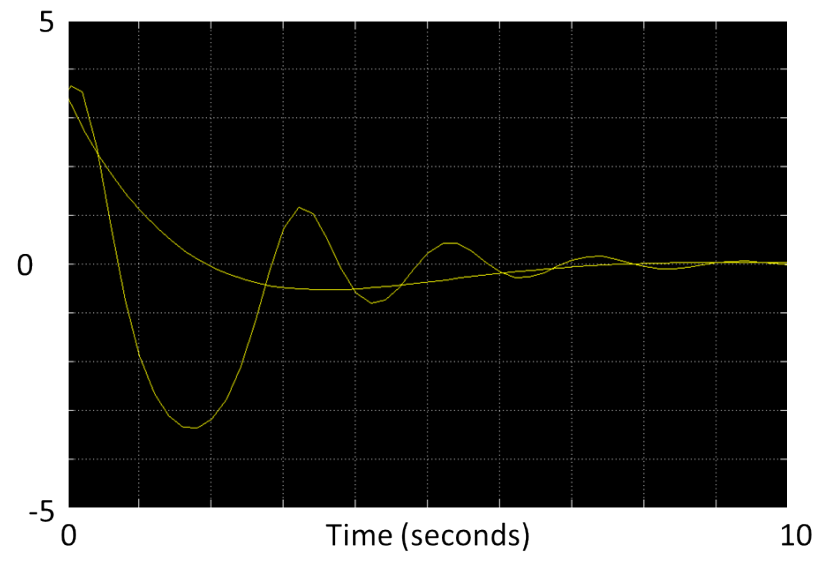

Figure 2: System Error Response: Oscillatory response with standard adaptive law and non-oscillatory response with the new adaptive law.

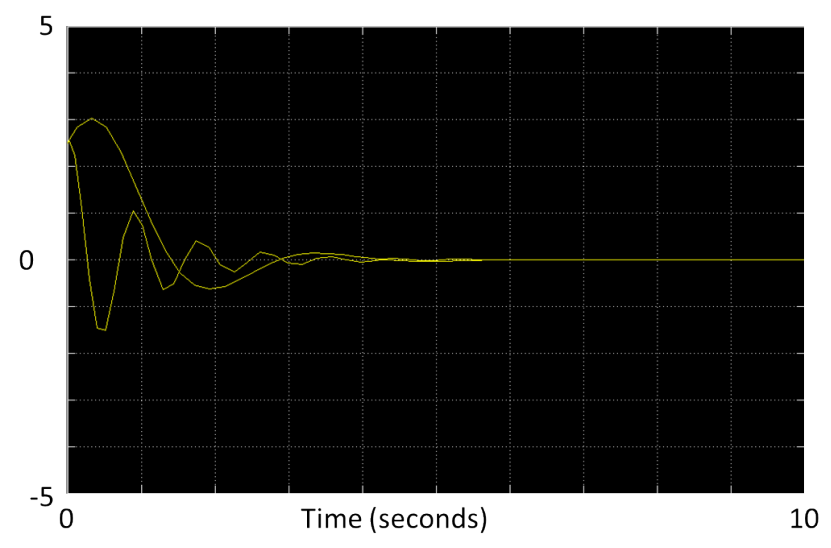

Figure 3: System Error Response: Oscillatory response with standard adaptive law and non-oscillatory response with the new adaptive law.

adaptive law, by choosing new positive definite quadratic Lyapunov function, given by

$$
V_{n 1}=\frac{1}{2}\left[e_{1}^{2}\right]+\frac{1}{2}\left[\frac{1}{g a_{2}}\right]\left[\phi^{2}\right]
$$

where gis a positive constant (say $g=1$ ) and A2 is a variable defined as

$$
\mathrm{A}_{2}=\int \mathrm{e}_{1}^{2} \cdot d t \text { leading to } \frac{d A_{2}}{d t}=e_{1}^{2} \text {. }
$$

Then, this function $V_{n 1}$ is differentiated with respect to time, system error equation is substituted and a new adaptive law is chosen as

$$
\dot{\phi}=-\left[g A_{2}\right] e_{1} y_{p}
$$

and substituted so that the differential of $V_{n}$ becomes as,

$$
\dot{V}_{n 1}=-a_{m} e_{1}^{2}-\frac{1}{2 g}\left[\frac{1}{A_{2}}\right]^{2} e_{1}^{2} \phi^{2}<0
$$

Now, for this new improved adaptive law [4] Eqn. (7), stability is assured as follows:since the $V_{n}$ is chosen as positive definite and the derivative of Vnis obtained as negative definite as long as $\left|e_{1}\right|$ is not zeros, the error system defined by the system output error equation and 
the adaptive law is uniformly asymptotically stable in the large (u.a.s.l.). For details of uniformly stable and uniformly asymptotically stable in the large (u.a.s.l.), see Narendra and Annaswamy [3].

\section{MRAC scheme for a $2^{\text {nd }}$ order plant}

For ease of exposition, consider the MRAC scheme with the controller a simple feedforward gain parameter. $k$; the reference model be a second order system with a gain $k_{m}$; and the plant is a second order system with a gain $k_{p}:$

$$
\begin{aligned}
& \text { Plant: }\left[s^{2}+a_{m 1} s+a_{m 0}\right] y_{p}=\left[k_{p}\right] u \text { with } u=k r \\
& \text { Reference Model: }\left[s^{2}+a_{m 1} s+a_{m 0}\right] y_{m}=\left[k_{m}\right] r
\end{aligned}
$$

wheres is the differential operator Let the system output error $e_{1}$ be defined as,

$$
e_{1}=y_{p}-y_{m} \text {. }
$$

Then, for the ideal controller parameter $k^{*}$, the plant controller combination becomes the reference model. For non-ideal values of $k$, there exists a parameter error, say, $\psi$ given by $\psi=k_{p} k-k m$. In this scenario, the design of MRAC anddevelopment of adaptive law for adjusting the $k$ based on Lyapunov stability theory can be outlined as follows [5-7].

The system output error equation can be written as

$$
\left[s^{2}+a_{m 1} s+a_{m 0}\right] e_{1}=[\psi] r \text { or } A_{m} e=\left[\begin{array}{ll}
0 & \psi r
\end{array}\right]^{T}
$$

In matrix form, $\mathrm{e}=\left[\mathrm{e}_{1} \mathrm{se}_{1}\right]$ and $A_{m}=\left[\begin{array}{cr}0 & 1 \\ -a m 1-a m 2\end{array}\right]$ so defined. Then, for the standard adaptive law, the positive definite quadratic Lyapunov function is chosen as

$$
V=e^{T} P e+\frac{1}{2}\left(\frac{\psi}{g}\right)^{2}
$$

Where the Lyapunov equation [8]

$$
A_{m}^{T} P+P A^{T}=-Q
$$

defines the $\mathrm{P}$ and $\mathrm{Q}$ as positive definite symmetric matrices. This function $V$ is differentiated with respect to time, system error equation is substituted and the standard adaptive law is chosen as

$$
\dot{\psi}=\dot{k}=-[g] e_{1} r \text { so that the derivative of V becomes, } \dot{V}=-e^{T} Q e \leq 0
$$

where gis a positive constant (say $g=1$ ). Now, for this standard adaptive law, stability is assured as follows: since the $V$ is chosen as positive definite and the derivative of $V$ is obtained as negative semi-definite, the error system defined by the system output error equation and the adaptive law is uniformly stable. For details, see Narendra and Annaswamy [3].

Where as the work in this paper presents a new improved standard adaptive law, by choosing new positive definite quadratic Lyapunov function, given by

$$
V_{n 2}=e^{T} P e+\frac{1}{2}\left[\frac{1}{g A_{2}}\right]\left[\psi^{2}\right]
$$

where gis a positive constant ( follows.

$$
\text { A2 }=\int \mathrm{e}_{1}^{2} \text {.dt leading to } \frac{d A_{2}}{d t}=e_{1}^{2} \text {. }
$$

Then, this function $V_{n 2}$ is differentiated with respect to time, system error equation is substituted and a new adaptive law is chosen as

$$
\begin{aligned}
& \dot{\psi}=\dot{k}=-g\left[A_{2}\right] e_{1} r \\
& \text { so that } \dot{V}_{n 2}=-e^{T} Q e-\frac{1}{2 g}\left[\frac{1}{A_{2}}\right]^{2} e_{1}^{2} \psi^{2}<0
\end{aligned}
$$

Now, for this new improved adaptive law Eqn. (16), stability is assured as follows:since the $V_{n 2}$ is chosen as positive definite and the derivative of $V_{n 2}$ is obtained as negative definite as long as $\left|e_{1}\right|$ is not zero, the error system defined by the system output error equation (11) and the adaptive law (16) is uniformly asymptotically stable in the large (u.a.s.l.). For details of uniformly stable and uniformly asymptotically stable in the large (u.a.s.l.), see Narendra and Annaswamy [3].

\section{Simulation Study Using Matlab Package}

A simulation study is carried on a 1st order plant with an MRAC schemegiven in Example 1 as in Section 2; and on a $2^{\text {nd }}$ order plant with an MRAC scheme given in Example 2 as in Section 3.

Example 1:The reference model transfer function is $\mathrm{Wm}=1 /(\mathrm{s}+1)$. The plant transfer function is $\mathrm{Wp}=1 /[\mathrm{s}+1]$. The feedback law isyp . The reference input is a step of magnitude 5 units.

Example 2: The reference model is chosen with a transfer function $\mathrm{Wm}=2 /\left(\mathrm{s}^{\wedge} 2+2 \mathrm{~s}+2\right)$. The plant is chosen with transfer function $\mathrm{Wp}=$ $\mathrm{Wm}$. The initial value of system error $\mathrm{e}(0)=2.5$. The adaptive laws used are: 1) Standard law with $g=1$ and 2) New law with $g=1$ and $0.01 \mathrm{~A} 2$.

\section{Conclusion}

New adaptive laws for Model Reference Adaptive Control (MRAC) systems have been developed. These new adaptive laws are based on the Lyapunov stability theory, as in many earlier papers on MRAC schemes. But, what is new is that derivative of the Lyapunov function is negative definite, as long as the system error is not zero; in addition, the adaptive gains are chosen directly from the integral of the square of the system error signal.The simulation study on $1^{\text {st }}$ and $2^{\text {nd }}$ order systems demonstrates that these new adaptive laws are better than the earlier adaptive laws for MRAC systems.

\section{References}

1. Ioannou PA, Sun J(1996) Stable and robust adaptive control. Prentice Hall, New Jersey, USA.

2. Rao MPRV (1998) Non-quadratic Lyapunov function and adaptive laws for MRAC. IEE Electronics Letters, 34: 2278 - 2280.

3. Narendra KS, Annaswamy AM (1989) Stable Adaptive Systems. Prentice Hall, Englewood Cliffs, New Jersey, USA.

4. Rao MPRV, Watters I (1999) Identification with non-quadratic Lyapunov function and new adaptive laws.IEE Electronics Letters, 35: 175-177.

5. RaoMPRV, UnglertM (2000) New Lyapunov function and new adaptive laws with e5-signal for MRAC. International Conference on Process Control and Instrumentation, Strathclyde University, Glasgow, Scotland, UK.

6. Spiegel MR (1969) Theory and problems of real variables: Lebesgue measure and integration. McGraw Hill Book Co, New York, USA.

7. Hackloth B, Butchart RT (1965) Synthesis of model reference adaptive systems by Lyapunov's second method. Proceedings of IFAC Symposium on the Theory of Self-Adaptive Control Systems. Teddington. England.

8. Donnelly DN (2002) Robust adaptive control using non-quadratic Lyapunov functions M.Sc Dissertation. University of Ulster, Jordanstown, Belfast, UK. 